\title{
Studied Sections
}

We investigated the Upper Barremian-Lower Aptian interval recovered at two drill sites: the Cismon core and DSDP Site 463 in the Mid-Pacific Mountains.

The Cismon drill site is located in the Southern Alps (north-eastern Italy) (46 $02^{\prime} \mathrm{N} ; 11^{\circ} 45^{\prime} \mathrm{E} ; 398 \mathrm{~m}$ altitude). The site was located on the continental margin of the Mesozoic Tethys, on the eastward deepening slope between the Trento Plateau and the Belluno Basin (Fig. DR1). The Cismon sequence was deposited at an estimated paleo-depth of 1000-1500 m during the Early Cretaceous (Weissert and Lini, 1991; Erba and Larson 1998; Bernoulli and Jenkyns, 2009). The Selli level is represented by a $\sim 5 \mathrm{~m}$-thick layer of hemipelagic sediments, between 23.67 and 18.64 stratigraphic meter depths (Erba and Larson, 1998; Erba et al., 1999). Lithologically the Selli level is characterized by marlstone alternating with black shales and discrete radiolarian beds (Erba et al., 1999).

DSDP Site 463 was drilled at a water depth of $2525 \mathrm{~m}$ in the ancient structural high of the western Mid-Pacific Mountains $\left(21^{\circ} 21.01^{\prime} \mathrm{N}, 174^{\circ} 40.07^{\prime} \mathrm{E}\right)$. During the Early Cretaceous, Site 463 was located at a paleo-latitude of $\sim 20^{\circ} \mathrm{S}$ (Fig. DR1), with a paleo-depth between a few hundred meters (Mélières et al., 1978 ) and $\sim 1 \mathrm{~km}$ (Roth, 1981). The Selli level at DSDP Site 463 is located between $\sim 626-615 \mathrm{mbsf}$, corresponding to $\sim 12 \mathrm{~m}$ of tuffaceous limestone containing a number of discrete organic-rich horizons with TOC up to 7 (wt\%) (Thiede et al., 1981; Erba, 1994).

\section{Methods}

We selected for analysis 27 samples from the Cismon core and 32 samples from DSDP Site 463 from an interval covering the Upper Barremian-Lower Aptian. We chose, where possible, black shale layers or organic rich samples (on the basis of their TOC content).

\section{1. $\underline{\text { Re-Os isotope analyses }}$}

Rhenium and osmium abundance data and Os-isotope compositions were determined on $\sim 0.5 \mathrm{~g}$ aliquots of powdered rock; analyses were performed at The Open University. Initial digestion took place using $12 \mathrm{ml}$ of inverse aqua regia $\left(3: 1\right.$ mixture of conc. $\mathrm{HNO}_{3}$ and conc. $\left.\mathrm{HCl}\right)$ in sealed Carius tubes at $180^{\circ} \mathrm{C}$ for 5 days, broadly following the methods of Cohen and Waters (1996).

Os was extracted into $\mathrm{CCl}_{4}$ (Cohen and Waters, 1996), and Re (in inverse aqua regia) was extracted into isoamylol. From the $\mathrm{CCl}_{4}$, Os was back extracted into $\mathrm{HBr}$ and purified by micro-distillation (following Roy-Barman and Allegre, 1995) using $\mathrm{CrO}_{3}$ in $\mathrm{H}_{2} \mathrm{SO}_{4}$ as oxidizing agent. Re and Os isotope ratio and abundance determinations were performed on a Thermo-Finnigan Triton high resolution multicollector mass spectrometer operated in N-TIMS mode (Cohen and Waters, 1996). 
Each batch of samples was analysed along with aliquots of an in-house mudrock standard and with appropriate Re and Os solution standards. Repeat measurements of a DTM Os solution standard yielded long-term ${ }^{187} \mathrm{Os} /{ }^{188} \mathrm{Os}$ reproducibility $<0.2 \%$ (2 s.d.), with a mean ${ }^{187} \mathrm{Os} /{ }^{188} \mathrm{Os}=0.17390 \pm 0.00014(\mathrm{n}=$ 32 ) over the period when the sample measurements were made.

All data were corrected for procedural blanks, amounting to $0.3-0.68 \mathrm{ppt}$ for Os and $3.8 \mathrm{ppt}$ for Re. The Os-isotope composition of seawater $\left({ }^{187} \mathrm{Os} /{ }^{188} \mathrm{Os}\right)$ was calculated from the analyses of individual samples assuming the Re-Os system became closed soon after sediment deposition (Tables DR1, DR2, DR3). Re-Os isotope regressions were calculated using Isoplot3.0 (Ludwig, 1998).

The initial Os-isotope ratios $\left({ }^{187} \mathrm{Os} /{ }^{188} \mathrm{Os}_{(\mathrm{i})}\right)$ were calculated assuming an age of $120 \mathrm{Ma}$ for the Selli interval, based on cyclochronology for the Cismon core (Malinverno et al., 2010). We note that an age of $125 \mathrm{Ma}$ was used by Tejada et al. (2009) for the samples that they analysed from Gorgo a Cerbara. The discrepancy in ${ }^{187} \mathrm{Os} /{ }^{188} \mathrm{Os}_{(\mathrm{i})}$ is, however, negligible (as indicated in Table DR5) when comparing the calculated values.

The exceptionally unradiogenic Os isotope compositions that characterise segment D (Fig. 1) are fully consistent with a large additional flux of unradiogenic Os from the hydrothermal alteration of OJP basalts. We assume a total volume for the OJP of $2 \times 10^{7} \mathrm{~km}^{3}$, with an Os concentration of $50 \mathrm{ppt}$ and a rock density of $3 \mathrm{~g} / \mathrm{cm}^{3}$ (note that the Os abundance in pristine OJP lavas is relatively high, with measured values between 20 and $160 \mathrm{ppt}-$ I. J. Parkinson, pers. comm.). The total mass of basalt emplaced was $6 \times 10^{19} \mathrm{~kg}$ and the total Os inventory in this basalt would have been $3 \times 10^{9} \mathrm{~kg}$, which is well over 2 orders of magnitude greater than the estimated present-day seawater Os budget of $1.4 \times 10^{7} \mathrm{~kg}$.

\section{Elemental analyses}

The concentrations of total organic carbon (TOC) wt $\%$, calcium carbonate $\left(\mathrm{CaCO}_{3}\right) \mathrm{wt} \%$, total nitrogen $(\mathrm{N})$ and total sulphur $(\mathrm{S})$ were determined on aliquots of the Cismon samples (Table DR2) using a LECO Instruments CNS-2000 elemental analyser at The Open University.

\section{Carbon stable-isotope analyses}

Carbon stable-isotope analyses were performed at Oxford University on bulk carbonate and organiccarbon fraction of 57 samples from DSDP Site 463. Long-term reproducibility, as determined from repeat measurements of our working standard, resulted in analytical uncertainties of $\delta^{13} \mathrm{C}_{\text {carb }}=2.09 \pm 0.07, \delta^{13}$ $\mathrm{C}_{\text {org }}=-26.91 \pm 0.11(\mathrm{n}=16)$; see Table DR4.

The values are reported in the conventional delta notation with respect to the Vienna Pee Dee Belemnite (V-PDB) standard (1):

$$
\delta^{13} \mathrm{C}_{\text {sample }}=\left\{\left[\left({ }^{13} \mathrm{C} /{ }^{12} \mathrm{C}\right)_{\text {sample }} /\left({ }^{13} \mathrm{C} /{ }^{12} \mathrm{C}\right)_{\text {reference }}\right]-1\right\} * 1000
$$


Analyses on the organic carbon fraction were performed on $2 \mathrm{~g}$ of powdered sample after sample decarbonation in $3 \mathrm{M} \mathrm{HCl}$.

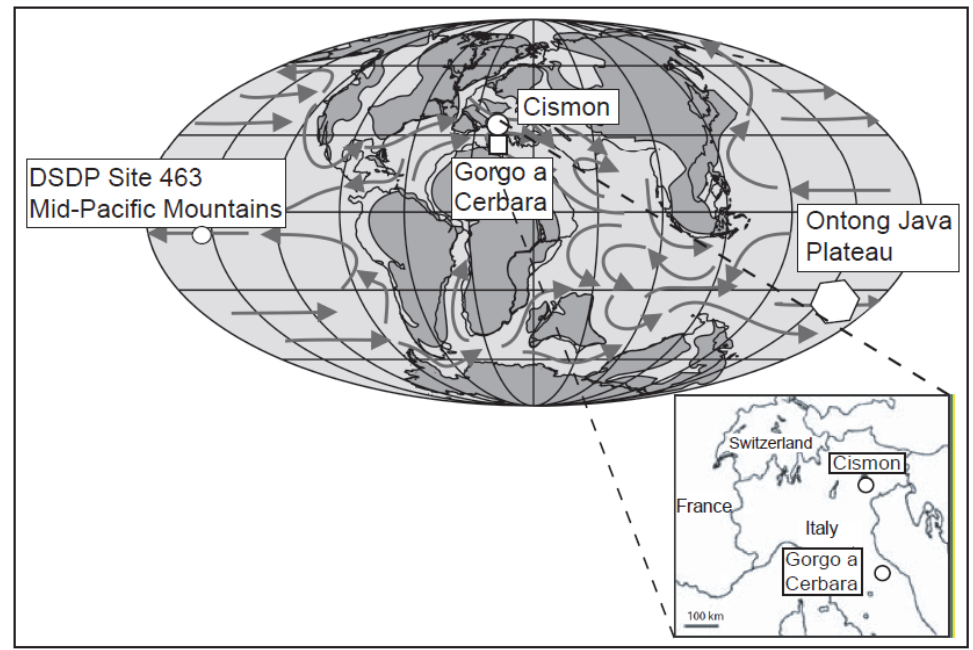

Figure DR1. Palaeogeographic reconstruction and proposed oceanic circulation at $100 \mathrm{Ma}$ (Hay, 2009). Location of DSDP Site 463 (Mid-Pacific Mountains), the Cismon drill site, and the Gorgo a Cerbara section are shown in relation to the Ontong Java Plateau. 


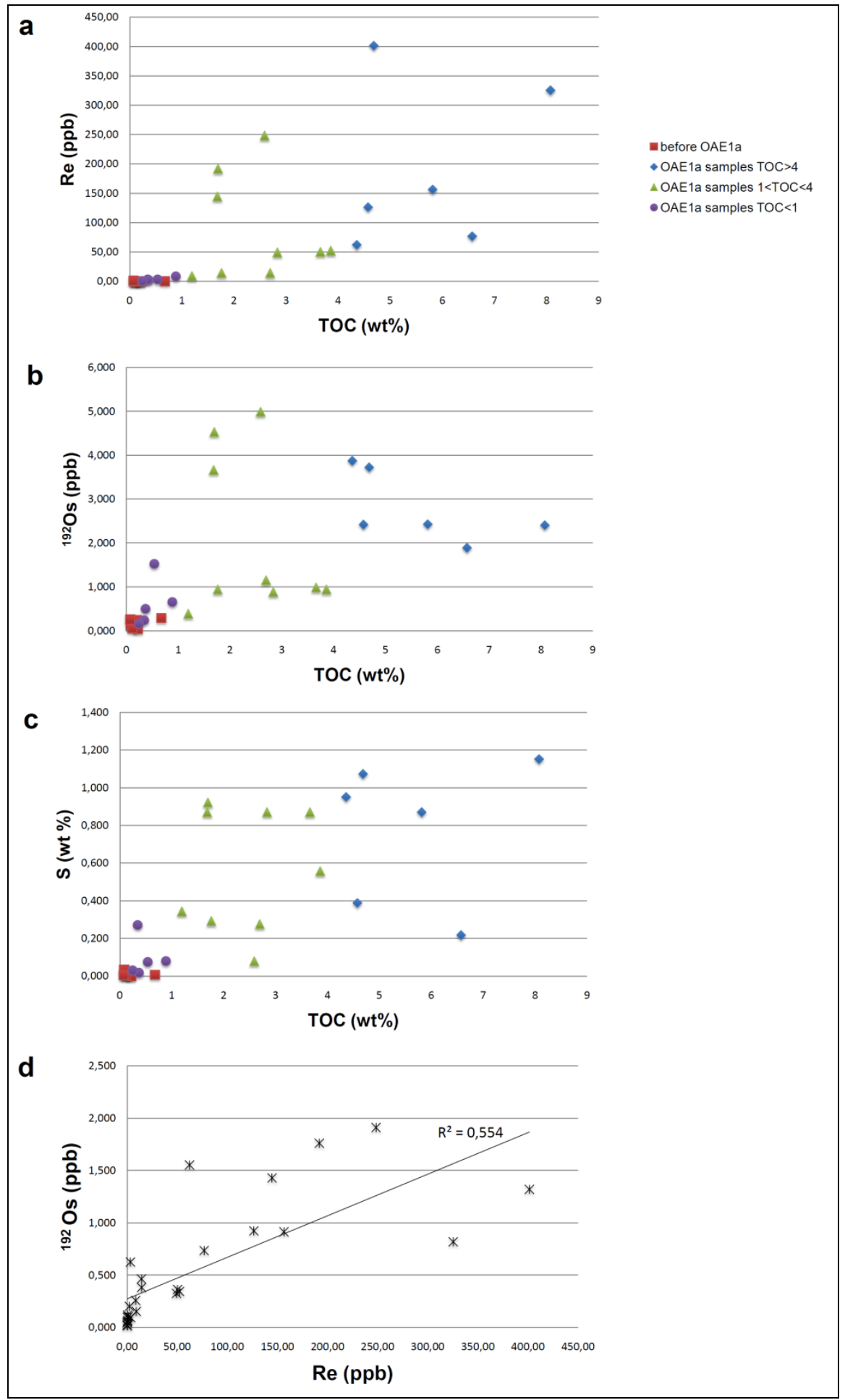

Figure DR2. Variation diagrams showing relationships between (a) TOC and Re concentrations, (b) TOC and ${ }^{192}$ Os concentrations, (c) S and TOC concentrations and (d) Re and ${ }^{192}$ Os concentrations, in samples from the Cismon core analysed in this study. In all cases there is a broad but sometimes very poorly defined positive relationship between the parameters displayed. 


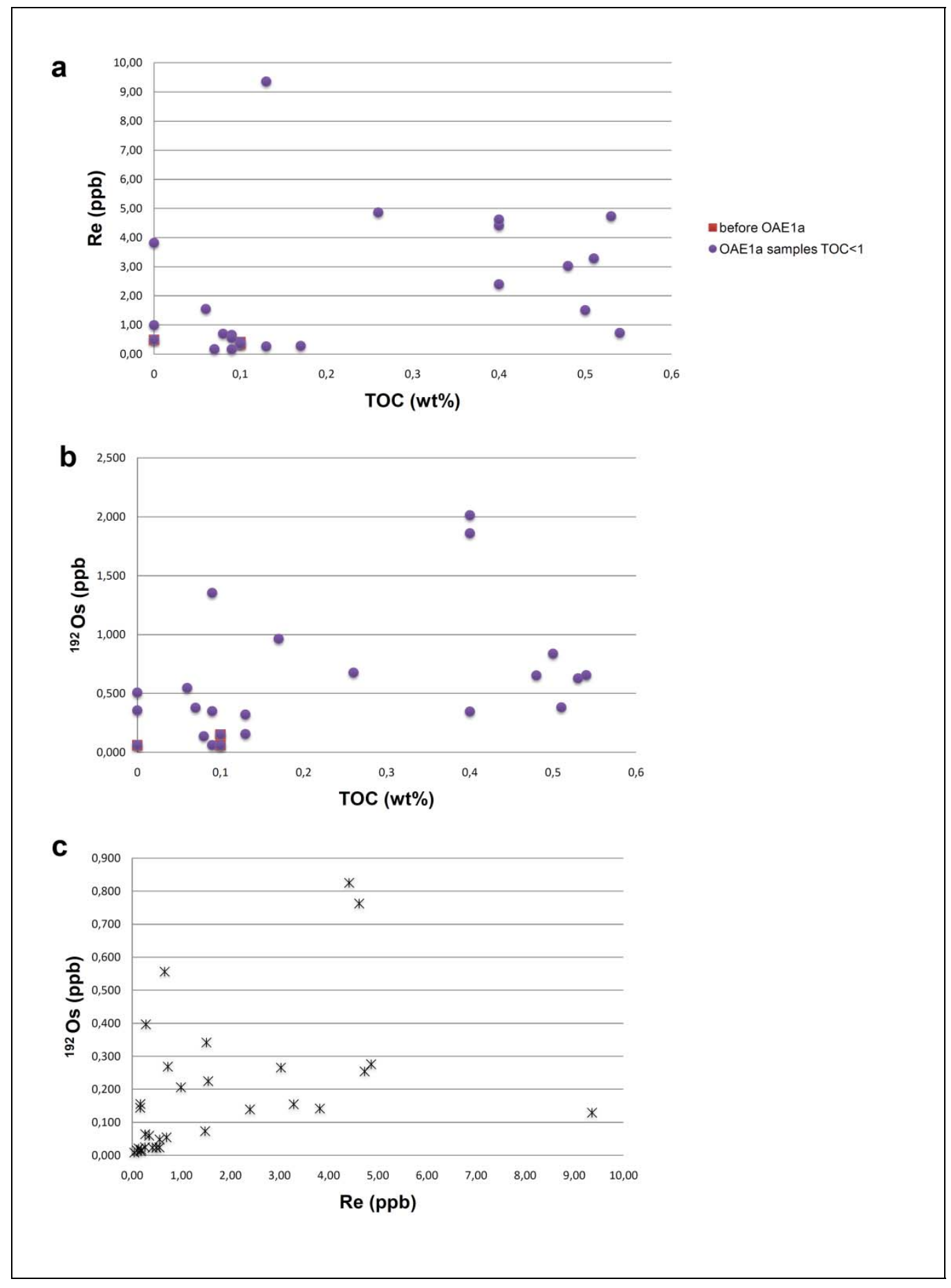

Figure DR3. Variation diagrams showing relationships between (a) TOC and Re concentrations, (b) TOC and ${ }^{192}$ Os concentrations, and (c) Re and ${ }^{192}$ Os concentrations, in samples from DSDP Site 463 analysed in this study. There are no clearly defined relationships between these parameters, although the data show broad positive correlations. 


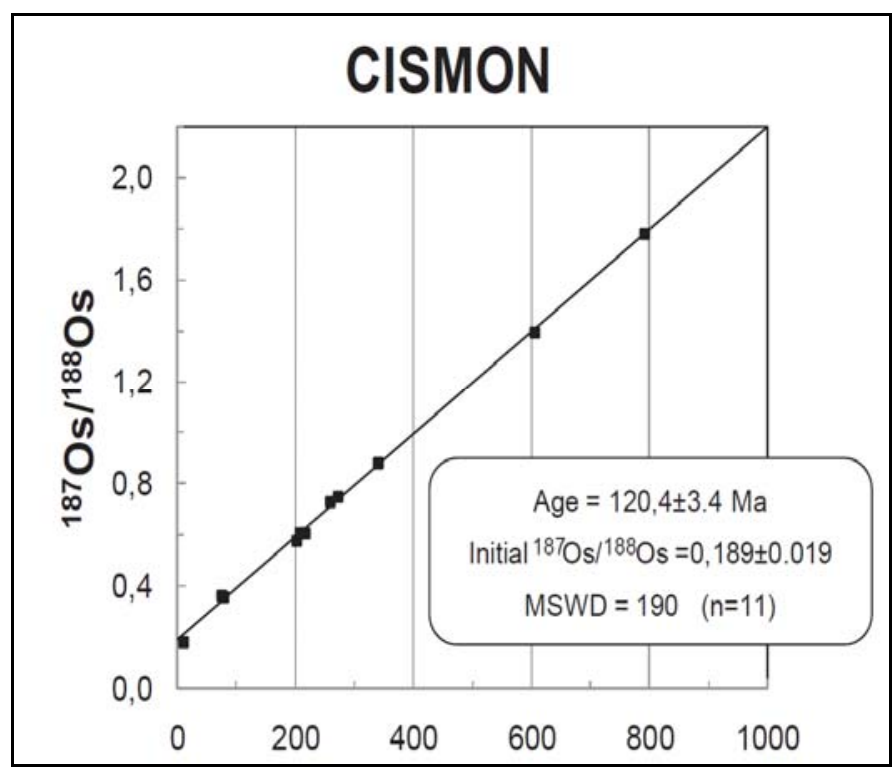

Figure DR4. Re-Os isochron evolution diagram with data from samples from Cismon core.

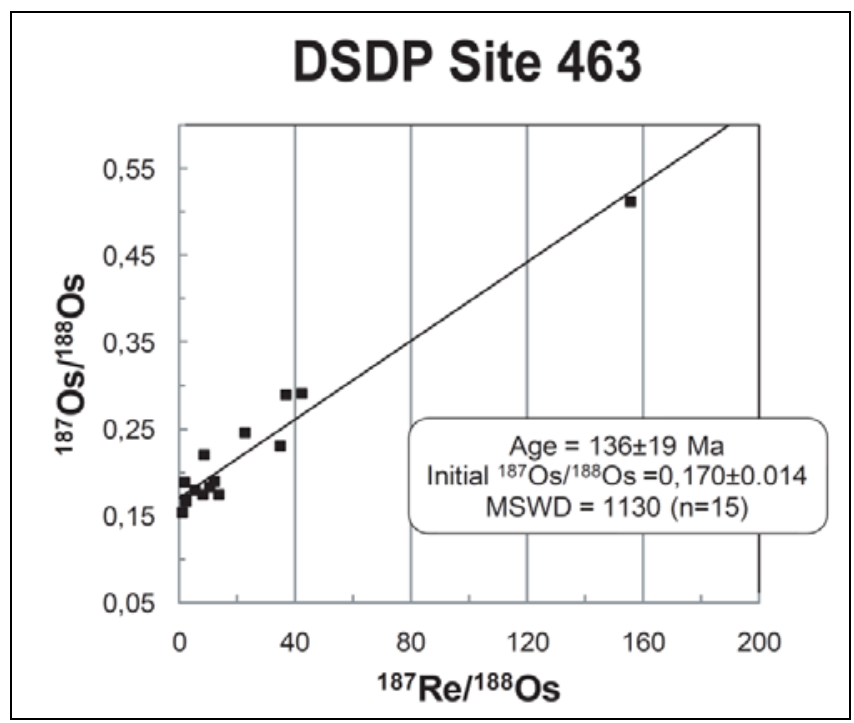

Figure DR5. Re-Os isochron evolution diagram with data from samples from DSDP Site 463. 


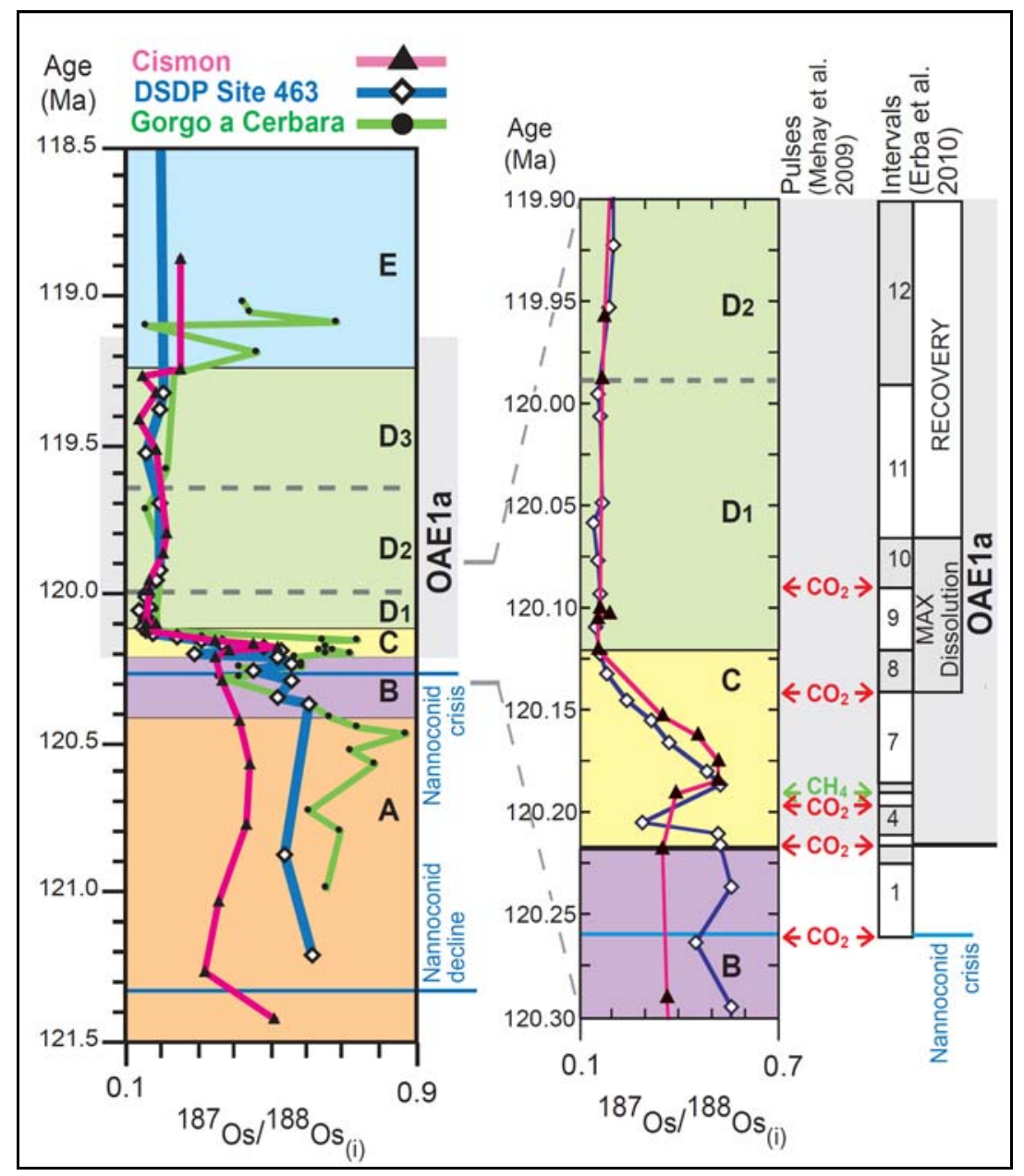

Figure DR6. Correlation between DSDP Site 463, Cismon and Gorgo a Cerbara (Tejada et al., 2009). On the right, close-up of the OAE 1a onset; Os-isotope data are correlated with the $\mathrm{CO}_{2}$ pulses (Méhay et al., 2009) and intervals of Erba et al. (2010). 


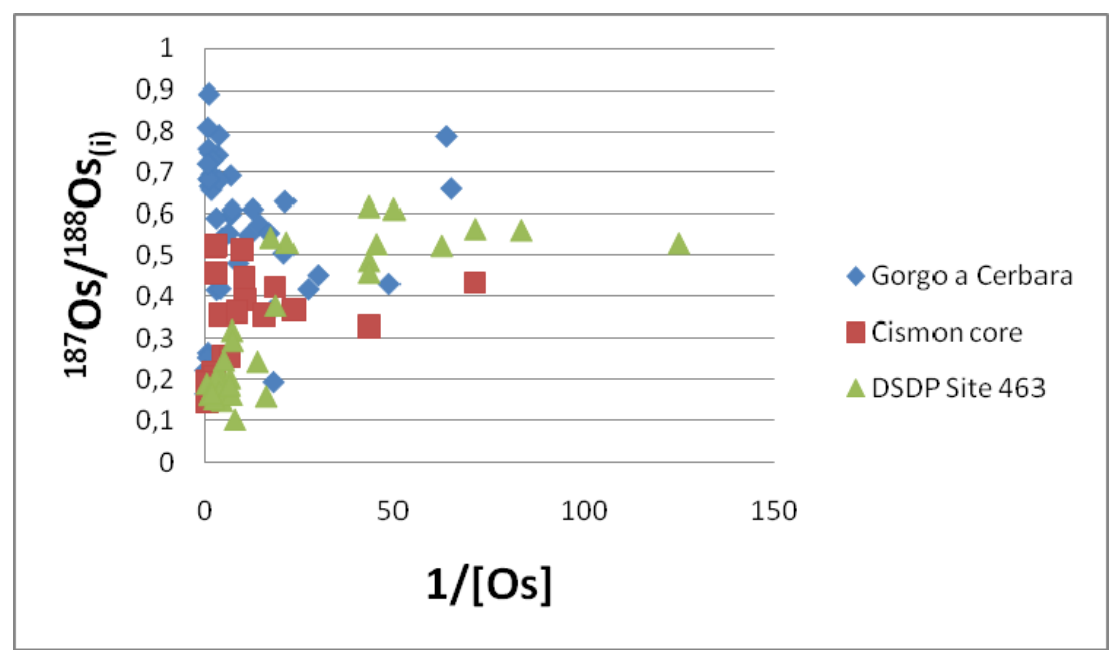

Figure DR7. Isotope mixing diagram showing the initial Os-isotope composition against the reciprocal of the Os abundance for samples from the Cismon core and DSDP Site 463 (this study), and for samples from Gorgo a Cerbara (Tejada et al., 2009).

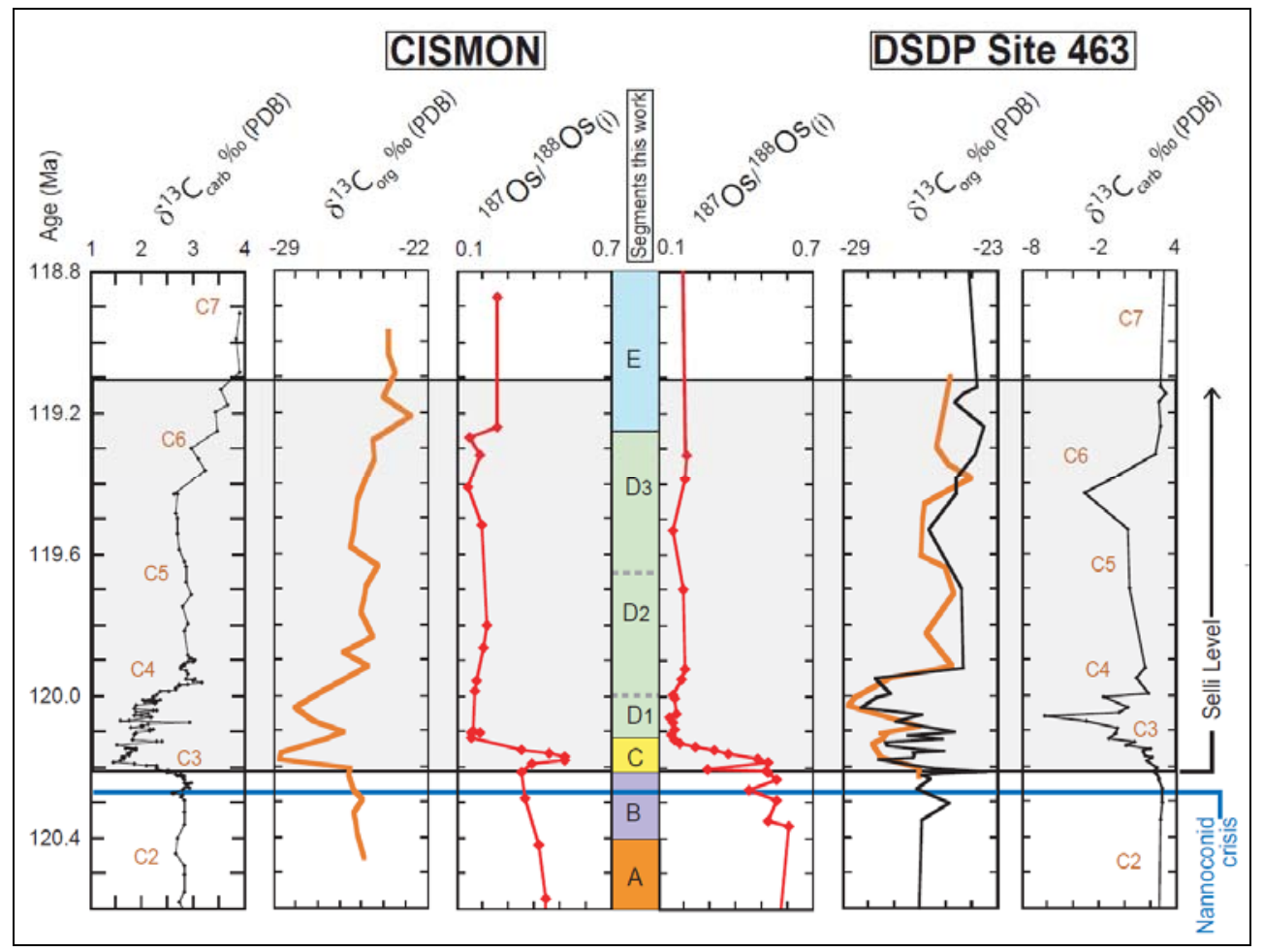

Figure DR8. Stratigraphic correlation between the Cismon core and DSDP Site 463. The correlation is based on $\delta^{13} \mathrm{C}_{\text {org }}$ values (orange) (Van Breugel et al., 2007) and $\delta^{13} \mathrm{C}_{\text {org }}$ values (black) from this study. $\delta^{13} \mathrm{C}_{\text {carb }}$ values for Cismon are from Erba et al. (2010); $\delta^{13} \mathrm{C}_{\text {carb }}$ values for DSDP Site 463 samples are from this study. The Os-isotope records are shown in red (this study). 


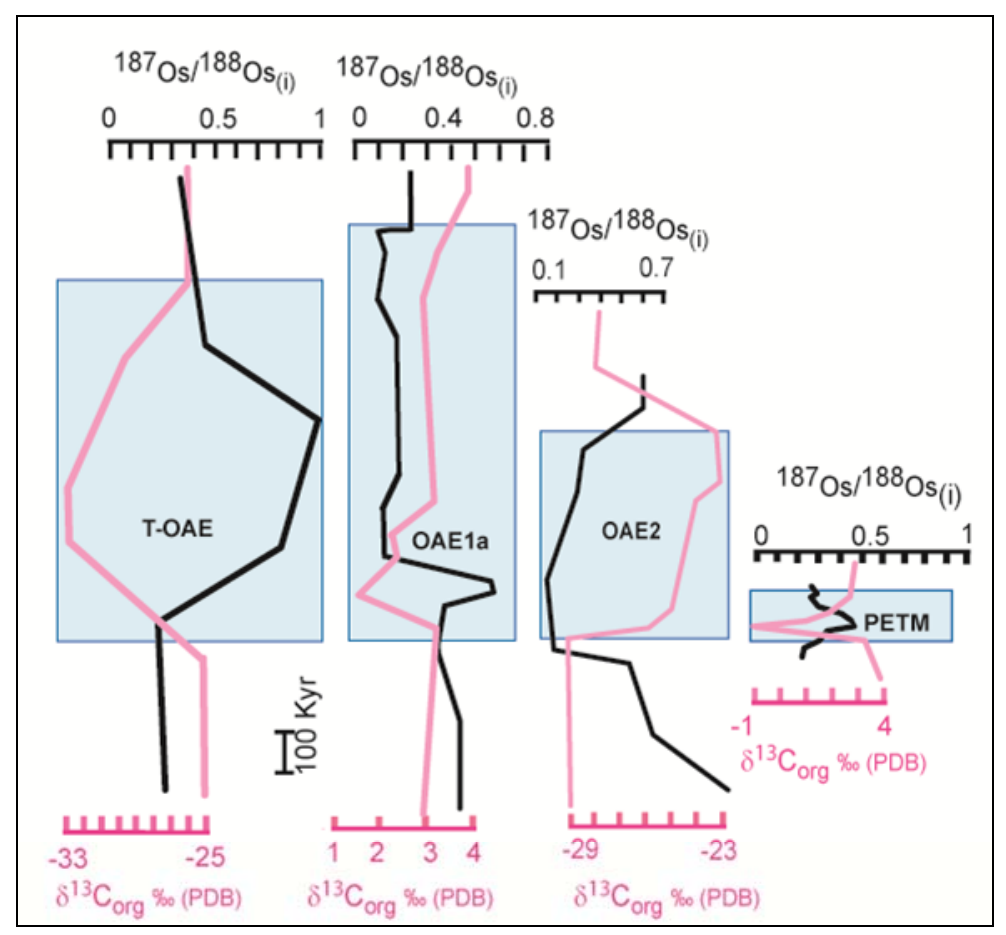

Figure DR9. Os-isotope data against $\delta^{13} \mathrm{C}$ for: The Toarcian OAE (T-OAE) (Cohen et al., 2004); OAE 1a (Erba et al., 2010; this study); OAE 2 (Turgeon and Creaser, 2008); and the PETM (Ravizza et al., 2001). Durations are respectively from Suan et al. (2008), Malinverno et al. (2010), Voigt et al. (2008) and Zachos et al. (2005).

Table DR1. Repeat ${ }^{187} \mathrm{Os} /{ }^{188}$ Os analyses.

\begin{tabular}{|c|c|c|c|c|c|c|c|}
\hline Sample & Age $(\mathrm{Ma})$ & $\operatorname{Re}(p p b)$ & Os (ppb) & ${ }^{187} \mathrm{Re} /{ }^{188} \mathrm{Os}$ & ${ }^{187} \mathrm{Os} /{ }^{188} \mathrm{Os}$ & $2 \sigma$ error & ${ }^{187} \mathrm{Os} /{ }^{188} \mathrm{Os}(\mathrm{i})$ \\
\hline 160_1 & 119.86 & 14.180 & 0.902 & 78.0 & 0.36 & 0.0002 & 0.207 \\
\hline $160 \_2$ & 119.86 & 14.640 & 0.924 & 78.6 & 0.36 & 0.0003 & 0.207 \\
\hline $160 \_3$ & 119.86 & 14.450 & 0.907 & 79.1 & 0.37 & 0.0007 & 0.209 \\
\hline $160 \_4$ & 119.86 & 14.314 & 0.909 & 78.2 & 0.36 & 0.0005 & 0.208 \\
\hline & & & & & & & \\
\hline $7 a \_1$ & 120.09 & 0.180 & 0.353 & 2.5 & 0.17 & 0.0011 & 0.162 \\
\hline $7 a \_2$ & 120.09 & 0.163 & 0.337 & 2.3 & 0.17 & 0.0003 & 0.161 \\
\hline & & & & & & & \\
\hline $5 a \_1$ & 120.12 & 0.350 & 1.318 & 1.3 & 0.17 & 0.0002 & 0.167 \\
\hline $5 a \_2$ & 120.12 & 0.660 & 1.307 & 2.4 & 0.17 & 0.0001 & 0.164 \\
\hline
\end{tabular}


Table DR2. Data for Cismon core samples showing their stratigraphic depth (m), calculated age (Ma) (following Malinverno et al., 2010), Re-Os isotope data and elemental abundance data.

\begin{tabular}{|c|c|c|c|c|c|c|c|c|c|c|c|c|c|c|}
\hline $\begin{array}{c}\text { Strat. } \\
\text { depth }(\mathrm{m})\end{array}$ & Age (Ma) & $\begin{array}{l}\mathrm{CaCO}_{3} \\
(\mathrm{wt} \%)\end{array}$ & $\begin{array}{c}\text { TOC } \\
\text { (wt \%) }\end{array}$ & $\begin{array}{c}\mathbf{S} \\
(w t \%)\end{array}$ & $\underset{\text { (wt\%) }}{\mathrm{N}}$ & $\operatorname{Re}(\mathrm{ppb})$ & $2 \sigma$ error & $\begin{array}{c}\text { Os } \\
(\mathrm{ppb})\end{array}$ & $2 \sigma$ error & $\begin{array}{l}{ }^{192} \mathrm{Os} \\
\text { (ppb) }\end{array}$ & ${ }^{187} \mathrm{Re} /{ }^{188}$ Os & ${ }^{187} \mathrm{Os}^{188} \mathrm{OS}_{(\mathrm{i})}$ & ${ }^{187}$ Os/ ${ }^{188}$ Os & $2 \sigma$ error \\
\hline 17.35 & 118.876 & 46.45 & 0.37 & 0.02 & 0.02 & 2.260 & 0.0035 & 0.482 & $4.18 \mathrm{E}-07$ & 0.195 & 23.138 & 0.258 & 0.304 & 0.000418 \\
\hline 19.17 & 119.242 & 36.83 & 1.19 & 0.34 & 0.03 & 9.150 & 0.0171 & 0.373 & $6.68 \mathrm{E}-07$ & 0.147 & 124.058 & 0.257 & 0.504 & 0.000668 \\
\hline 19.29 & 119.271 & 34.27 & 8.08 & 1.15 & 0.28 & 325.500 & 1.8136 & 2.323 & $9.80 \mathrm{E}-07$ & 0.789 & 820.278 & 0.149 & 1.781 & 0.000980 \\
\hline 19.5 & 119.321 & 28.28 & 4.58 & 0.39 & 0.16 & 126.430 & 0.1150 & 2.337 & $5.81 \mathrm{E}-07$ & 0.893 & 281.692 & 0.189 & 0.749 & 0.000581 \\
\hline 19.87 & 119.411 & 35.55 & 4.69 & 1.07 & 0.17 & 401.500 & 0.6674 & 3.599 & $8.42 \mathrm{E}-07$ & 1.276 & 625.878 & 0.146 & 1.392 & 0.000842 \\
\hline 20.32 & 119.518 & 7.03 & 4.36 & 0.95 & 0.20 & 62.260 & 1.2485 & 3.739 & $2.79 \mathrm{E}-07$ & 1.499 & 82.598 & 0.195 & 0.360 & 0.000279 \\
\hline 21.59 & 119.800 & 33.94 & 2.70 & 0.28 & 0.11 & 14.280 & 0.0208 & \begin{tabular}{|l|}
1.112 \\
\end{tabular} & $2.31 \mathrm{E}-07$ & 0.447 & 63.560 & 0.216 & 0.343 & 0.000231 \\
\hline 21.9 & 119.864 & 38.48 & 1.76 & 0.29 & 0.06 & 14.400 & 0.0178 & 0.911 & $4.31 \mathrm{E}-07$ & 0.366 & 78.448 & 0.208 & 0.364 & 0.000431 \\
\hline 22.36 & 119.958 & 44.71 & 6.57 & 0.22 & 0.21 & 76.820 & 0.2753 & 1.827 & 9.67E-07 & 0.710 & 215.176 & 0.176 & 0.607 & 0.000967 \\
\hline 22.51 & 119.988 & 30.45 & 5.82 & 0.87 & 0.21 & 156.490 & 0.5523 & 2.345 & $3.83 \mathrm{E}-07$ & 0.882 & 352.812 & 0.172 & 0.878 & 0.000383 \\
\hline 23.08 & 120.101 & 15.06 & 1.68 & 0.08 & 0.06 & 144.620 & 1.4109 & \begin{tabular}{|l|}
3.538 \\
\end{tabular} & $4.50 \mathrm{E}-07$ & 1.381 & 208.414 & 0.161 & 0.579 & 0.000450 \\
\hline 23.09 & 120.103 & 14.42 & 2.59 & 0.92 & 0.10 & 248.440 & 0.5034 & 4.821 & $3.39 \mathrm{E}-07$ & 1.846 & 267.570 & 0.191 & 0.727 & 0.000339 \\
\hline 23.1 & 120.105 & 14.37 & 1.70 & 0.87 & 0.07 & 191.800 & 1.6394 & \begin{tabular}{|l|}
4.377 \\
\end{tabular} & 1.47E-06 & 1.701 & 224.229 & 0.158 & 0.607 & 0.001473 \\
\hline 23.18 & 120.121 & 14.04 & 0.54 & 0.08 & 0.02 & 3.200 & 0.0078 & 1.470 & 1.0 & 0.603 & 10.561 & 0.160 & 0.182 & 0108 \\
\hline 23.34 & 120.153 & 20.93 & 0.89 & 0.08 & 0.04 & 8.480 & 0.0090 & 0.631 & -07 & 0.249 & 67.764 & 0.356 & 0.492 & 0330 \\
\hline 23.39 & 120.163 & 17.54 & 2.84 & 0.56 & 0.12 & 49.270 & 0.0390 & \begin{tabular}{|l|}
0.852 \\
\end{tabular} & $4.61 \mathrm{E}-07$ & 0.313 & 313.380 & 0.457 & 1.085 & 0.000461 \\
\hline 23.45 & 120.175 & 16.59 & 3.66 & 0.74 & 0.17 & 50.310 & 0.0260 & 0.953 & 7.73E-07 & 0.350 & 286.290 & 0.520 & 1.094 & 0.000773 \\
\hline 23.5 & 120.185 & 24.10 & 3.86 & 0.77 & 0.17 & 52.250 & 0.0355 & 0.911 & $6.58 \mathrm{E}-07$ & 0.332 & 313.243 & 0.524 & 1.152 & 0.000658 \\
\hline 23.53 & 120.191 & 32.06 & 0.34 & 0.27 & 0.02 & 3.480 & 0.0049 & \begin{tabular}{|l|}
0.232 \\
\end{tabular} & $6.25 \mathrm{E}-07$ & 0.092 & 75.953 & 0.392 & 0.545 & 0.000625 \\
\hline 23.67 & 120.218 & 39.81 & 0.24 & 0.03 & 0.01 & 0.650 & 0.0018 & 0.157 & 4.24E-07 & 0.063 & 20.643 & 0.356 & 0.397 & 0.000424 \\
\hline 24.18 & 120.291 & 65.21 & 0.12 & 0.00 & 0.01 & 0.120 & 0.0012 & 0.104 & $4.61 \mathrm{E}-07$ & 0.042 & 5.608 & 0.368 & 0.379 & 0.000461 \\
\hline 25.10 & 120.422 & 50.07 & 0.08 & 0.03 & 0.01 & 0.290 & 0.0014 & \begin{tabular}{|l|}
0.131 \\
\end{tabular} & $4.02 \mathrm{E}-07$ & 0.053 & 11.234 & 0.423 & 0.446 & 0.000402 \\
\hline 26.18 & 120.575 & 42.16 & 0.20 & 0.00 & 0.04 & 0.280 & 0.0589 & \begin{tabular}{|l|}
0.232 \\
\end{tabular} & $6.91 \mathrm{E}-07$ & 0.093 & 6.036 & 0.446 & 0.458 & 0.000691 \\
\hline 27.59 & 120.777 & 87.13 & 0.22 & 0.00 & 0.03 & 0.290 & 0.0016 & \begin{tabular}{|l|}
0.035 \\
\end{tabular} & $1.62 \mathrm{E}-06$ & 0.014 & 40.839 & 0.434 & 0.517 & 0.001623 \\
\hline 29.39 & 121.029 & 28.08 & 0.68 & 0.01 & 0.05 & 0.370 & 0.0438 & 0.283 & $9.91 \mathrm{E}-07$ & 0.114 & 6.452 & 0.364 & 0.377 & 0.000991 \\
\hline 31.09 & 121.270 & 80.87 & 0.11 & 0.00 & 0.03 & 0.010 & 0.0021 & 0.055 & $2.70 \mathrm{E}-06$ & 0.023 & 1.320 & 0.328 & 0.330 & 0.002703 \\
\hline 32.43 & 121.424 & 25.81 & 0.07 & 0.01 & 0.05 & 1.120 & 0.9240 & 0.251 & $8.03 \mathrm{E}-07$ & 0.099 & 22.705 & 0.513 & 0.559 & 0.000803 \\
\hline
\end{tabular}

Table DR3. Data for DSDP Site 463 samples showing stratigraphic depth (m), calculated age (Ma) (following Malinverno et al., 2010), and Re-Os isotope data.

\begin{tabular}{|c|c|c|c|c|c|c|c|c|c|c|c|c|c|c|}
\hline Core & Section & $\mathrm{cm}$ & $\begin{array}{l}\text { Strat. } \\
\text { depth } \\
\text { (m) }\end{array}$ & $\begin{array}{c}\mathrm{N} \\
\text { sample }\end{array}$ & $\begin{array}{l}\text { Age } \\
(\mathrm{Ma})\end{array}$ & $\begin{array}{c}\mathrm{Re} \\
(\mathrm{ppb})\end{array}$ & $2 \sigma$ error & $\begin{array}{c}\text { Os } \\
(\mathrm{ppb})\end{array}$ & $2 \sigma$ error & $\begin{array}{l}{ }^{192} \mathrm{Os} \\
\text { (ppb) }\end{array}$ & ${ }^{187} \operatorname{Re} /{ }^{188}$ Os & ${ }^{187} \mathrm{Os} /{ }^{188} \mathrm{Os}_{(\mathrm{i})}$ & ${ }^{187}$ Os $/{ }^{188}$ Os & $2 \sigma$ error \\
\hline 69 & $\mathrm{CC}$ & $7-9$ & 607.57 & 33 & 118.05 & 1.48 & 0.0022 & 0.175 & 3.96E-07 & 0.071 & 41.888 & 0.242 & 0.324 & 0.000396 \\
\hline 70 & 1 & $125-126$ & 614.75 & 26 & 119.18 & 9.36 & 0.1806 & 0.309 & 7.12E-07 & 0.124 & 151.002 & 0.100 & 0.400 & 0.000712 \\
\hline 70 & 2 & $123-125$ & 616.23 & 18 & 119.32 & 4.73 & 0.0073 & 0.606 & $3.30 \mathrm{E}-07$ & 0.245 & 38.368 & 0.213 & 0.289 & 0.000330 \\
\hline 70 & 3 & $39-40$ & 616.89 & 14 & 119.39 & 3.29 & 0.0549 & 0.368 & 1.99E-06 & 0.149 & 43.949 & 0.202 & 0.290 & 0.001985 \\
\hline 70 & 3 & $140-141$ & 617.9 & 9 & 119.53 & 4.86 & 0.0831 & 0.653 & $3.82 \mathrm{E}-07$ & 0.266 & 36.362 & 0.157 & 0.230 & 0.000382 \\
\hline 70 & 4 & $80-82$ & 618.82 & 4 & 119.70 & 3.03 & 0.0034 & 0.630 & 1.48E-07 & 0.256 & 23.487 & 0.199 & 0.246 & 0.000148 \\
\hline 70 & 5 & $60-61$ & 620.1 & $25 a$ & 119.92 & 1.51 & 0.0529 & 0.808 & $2.48 \mathrm{E}-07$ & 0.330 & 9.104 & 0.202 & 0.220 & 0.000248 \\
\hline 70 & 5 & $123-124$ & 620.73 & $22 a$ & 119.95 & 142.85 & 0.1634 & 4.461 & $9.16 \mathrm{E}-07$ & 1.754 & 161.947 & 0.188 & 0.512 & 0.000916 \\
\hline 70 & 6 & $59-61$ & 621.59 & $17 a$ & 120.00 & 4.42 & 0.0073 & 1.941 & $4.50 \mathrm{E}-07$ & 0.796 & 11.033 & 0.160 & 0.183 & 0.000450 \\
\hline 70 & 6 & $81-82$ & 621.81 & $16 a$ & 120.01 & 4.62 & 0.0731 & 1.763 & $2.21 \mathrm{E}-07$ & 0.723 & 12.718 & 0.163 & 0.189 & 0.000221 \\
\hline 70 & 7 & $7-8$ & 622.57 & $12 a$ & 120.05 & 0.73 & 0.0091 & 0.632 & 4.47E-07 & 0.259 & 5.570 & 0.168 & 0.180 & 0447 \\
\hline 70 & 7 & $19-20$ & 622.69 & $11 a$ & 120.06 & 1.55 & 0.0155 & 0.528 & 4.69E-07 & 0.217 & 14.208 & 0.146 & 0.174 & 0.000469 \\
\hline 70 & $\mathrm{CC}$ & $5-6$ & 622.91 & $10 a$ & 120.08 & 0.27 & 0.0080 & 0.149 & 9.36E-05 & 0.061 & 8.622 & 0.157 & 0.175 & 0.093585 \\
\hline 71 & 1 & $16-18$ & 623.16 & $7 a$ & 120.09 & 0.16 & 0.0012 & 0.337 & $3.00 \mathrm{E}-07$ & 0.139 & 2.338 & 0.161 & 0.165 & 0.000300 \\
\hline 71 & 1 & $42-43$ & 623.42 & $6 a$ & 120.11 & 0.28 & 0.0100 & 0.950 & 1.01E-07 & 0.391 & 1.419 & 0.151 & 0.154 & 0.000101 \\
\hline 71 & 1 & $61-62$ & 623.61 & $5 a$ & 120.12 & 0.66 & 0.0065 & \begin{tabular}{|l|}
1.307 \\
\end{tabular} & 1.11E-07 & 0.537 & 2.444 & 0.164 & 0.169 & 0.000111 \\
\hline 71 & 1 & $76-78$ & 623.76 & $4 a$ & 120.13 & 0.17 & 0.0083 & 0.364 & $1.74 \mathrm{E}-07$ & 0.150 & 2.235 & 0.184 & 0.188 & 0.000174 \\
\hline 71 & 1 & $96-97$ & 623.96 & $3 a$ & 120.15 & 0.99 & 0.0097 & 0.481 & $2.16 \mathrm{E}-07$ & 0.195 & 10.122 & 0.245 & 0.265 & 0.000216 \\
\hline 71 & 1 & $124-125$ & 624.24 & $2 a$ & 120.16 & 3.82 & 0.0321 & 0.344 & 1.11E-06 & 0.137 & 55.515 & 0.317 & 0.429 & 0.001108 \\
\hline 71 & 2 & $1-3$ & 624.51 & $78 \mathrm{c}$ & 120.17 & 0.70 & 0.0023 & 0.131 & 4.27E-06 & 0.053 & 26.622 & 0.376 & 0.429 & 0.004267 \\
\hline 71 & 2 & $39-40$ & 624.89 & $76 \mathrm{c}$ & 120.18 & 0.56 & 0.0073 & 0.058 & 8.89E-07 & 0.023 & 48.453 & 0.485 & 0.582 & 0.000889 \\
\hline 71 & 2 & $58-59$ & 625.08 & $75 \mathrm{c}$ & 120.19 & 0.56 & 0.0083 & 0.118 & $8.61 \mathrm{E}-07$ & 0.046 & 24.068 & 0.530 & 0.579 & 0.000861 \\
\hline 71 & 2 & $78-80$ & 625.28 & $74 \mathrm{c}$ & 120.21 & 2.40 & 0.0035 & 0.334 & $3.33 \mathrm{E}-06$ & 0.134 & 35.607 & 0.293 & 0.365 & 0.003333 \\
\hline 71 & 2 & $100-102$ & 625.5 & $73 \mathrm{c}$ & 120.21 & 0.18 & 0.0075 & 0.041 & $2.19 \mathrm{E}-06$ & 0.016 & 22.717 & 0.522 & 0.567 & 0.002185 \\
\hline 71 & 2 & $120-121$ & 625.7 & $72 \mathrm{c}$ & 120.22 & 0.04 & 0.0032 & 0.019 & $8.11 \mathrm{E}-06$ & 0.008 & 11.035 & 0.528 & 0.550 & 0.008113 \\
\hline 71 & 3 & $43-46$ & 626.43 & $68 \mathrm{c}$ & 120.24 & 0.10 & 0.0050 & 0.035 & $2.05 E-06$ & 0.014 & 13.833 & 0.563 & 0.591 & 0.002051 \\
\hline 71 & 3 & $116-117$ & 627.16 & $64 \mathrm{c}$ & 120.26 & 0.26 & 0.0044 & 0.058 & $4.67 \mathrm{E}-06$ & 0.023 & 22.544 & 0.457 & 0.502 & 0.004667 \\
\hline 71 & 4 & $7-8$ & 627.57 & $62 \mathrm{c}$ & 120.29 & 0.18 & 0.0083 & 0.030 & 2.07E-06 & 0.012 & 30.484 & 0.560 & 0.621 & 0.002068 \\
\hline 71 & 4 & $85-87$ & 628.35 & $58 \mathrm{c}$ & 120.35 & 0.41 & 0.0117 & 0.057 & 6.61E-07 & 0.022 & 37.235 & 0.526 & 0.601 & 0.000661 \\
\hline 71 & $\mathrm{CC}$ & $7-8$ & 628.56 & $57 \mathrm{c}$ & 120.37 & 0.13 & 0.0063 & 0.051 & $1.29 \mathrm{E}-06$ & 0.020 & 13.111 & 0.611 & 0.637 & 0.001285 \\
\hline 72 & 2 & $141-142$ & 635.41 & $40 \mathrm{c}$ & 120.88 & 0.35 & 0.0035 & 0.146 & $1.62 \mathrm{E}-06$ & 0.057 & 12.082 & 0.542 & 0.567 & 0.001624 \\
\hline 73 & 2 & $0-3$ & 643.5 & $16 \mathrm{c}$ & 121.21 & 0.49 & 0.0017 & 0.058 & 4.39E-06 & 0.023 & 43.935 & 0.617 & 0.705 & 0.004390 \\
\hline
\end{tabular}


Table DR4. Carbon stable-isotope data for bulk carbonate and bulk organic-carbon fractions for samples from DSDP Site 463, against stratigraphic depth (m).

\begin{tabular}{|c|c|c|c|c|c|}
\hline CORE & SECTION & $\mathrm{cm}$ & m (corrected) & $\delta^{13} \mathrm{C}_{\text {carb }}(\% \circ \mathrm{PDB})$ & $\delta^{13} C_{\text {org }}(\% \circ$ PDB $)$ \\
\hline 69 & 2 & $105-106$ & 606.55 & 4.622 & \\
\hline 69 & 2 & $120-122$ & 606.7 & 4.527 & -24.63 \\
\hline 69 & 3 & $20-22$ & 607.2 & 4.546 & -24.15 \\
\hline 69 & 3 & $40-42$ & 607.4 & 4.388 & -24.57 \\
\hline 69 & $\mathrm{CC}$ & $7-9$ & 607.57 & 3.779 & -24.84 \\
\hline 70 & 1 & $77-79$ & 614.27 & 2.810 & -23.76 \\
\hline 70 & 1 & $96-98$ & 614.46 & 3.260 & -24.29 \\
\hline 70 & 1 & $125-126$ & 614.75 & 2.745 & -24.65 \\
\hline 70 & 2 & $40-42$ & 615.4 & 2.895 & -23.51 \\
\hline 70 & 2 & $123-125$ & 616.23 & 2.410 & -23.83 \\
\hline 70 & 3 & $39-40$ & 616.89 & -0.724 & -24.57 \\
\hline 70 & 3 & $85-87$ & 617.35 & -3.067 & -24.57 \\
\hline 70 & 3 & $140-141$ & 617.9 & 0.322 & -25.66 \\
\hline 70 & 4 & $80-82$ & 618.82 & 0.373 & -24.37 \\
\hline 70 & 5 & 60-61 & 620.1 & 1.674 & -24.3 \\
\hline 70 & 5 & 123-124 & 620.73 & 0.915 & -27.74 \\
\hline 70 & 6 & $59-61$ & 621.59 & 1.900 & -27.17 \\
\hline 70 & 6 & $81-82$ & 621.81 & -1.652 & -27.69 \\
\hline 70 & 6 & $141-142$ & 622.41 & 0.332 & -28.29 \\
\hline 70 & 7 & $7-8$ & 622.57 & -0.426 & -25.93 \\
\hline 70 & 7 & $19-20$ & 622.69 & -6.149 & -26.94 \\
\hline 70 & $\mathrm{CC}$ & $5-6$ & 622.91 & -2.983 & -24.66 \\
\hline 71 & 1 & $16-18$ & 623.16 & -0.478 & -26.46 \\
\hline 71 & 1 & $42-43$ & 623.42 & -0.669 & -25.15 \\
\hline 71 & 1 & $61-62$ & 623.61 & -1.228 & -27.33 \\
\hline 71 & 1 & $76-78$ & 623.76 & 0.879 & \\
\hline 71 & 1 & 96-97 & 623.96 & 0.191 & -27.3 \\
\hline 71 & 1 & 124-125 & 624.24 & 2.019 & -26.04 \\
\hline 71 & 1 & $138-141$ & 624.38 & 1.476 & -25.04 \\
\hline 71 & 2 & $1-3$ & 624.51 & 1.542 & -26.29 \\
\hline 71 & 2 & $19-20$ & 624.69 & 1.654 & -26.2 \\
\hline 71 & 2 & $39-40$ & 624.89 & 2.132 & -26.3 \\
\hline 71 & 2 & $58-59$ & 625.08 & 1.818 & -27.64 \\
\hline 71 & 2 & $78-80$ & 625.28 & 2.521 & -25.55 \\
\hline 71 & 2 & $120-121$ & 625.7 & 2.276 & -23.43 \\
\hline 71 & 2 & $140-142$ & 625.9 & 2.477 & -25.29 \\
\hline 71 & 3 & $0-3$ & 626.0 & 2.518 & -25.95 \\
\hline 71 & 3 & $43-46$ & 626.43 & 2.676 & -25.59 \\
\hline & & & 626.59 & 2.708 & \\
\hline 71 & 3 & $116-117$ & 627.16 & 3.035 & -26.11 \\
\hline 71 & 4 & $19-20$ & 627.69 & 2.938 & -24.85 \\
\hline 71 & 4 & $85-87$ & 628.35 & 2.909 & -25.9 \\
\hline 72 & 1 & $0-3$ & 632.5 & 2.704 & -26.04 \\
\hline 72 & 2 & $0-6$ & 634.0 & 3.252 & -26.18 \\
\hline
\end{tabular}




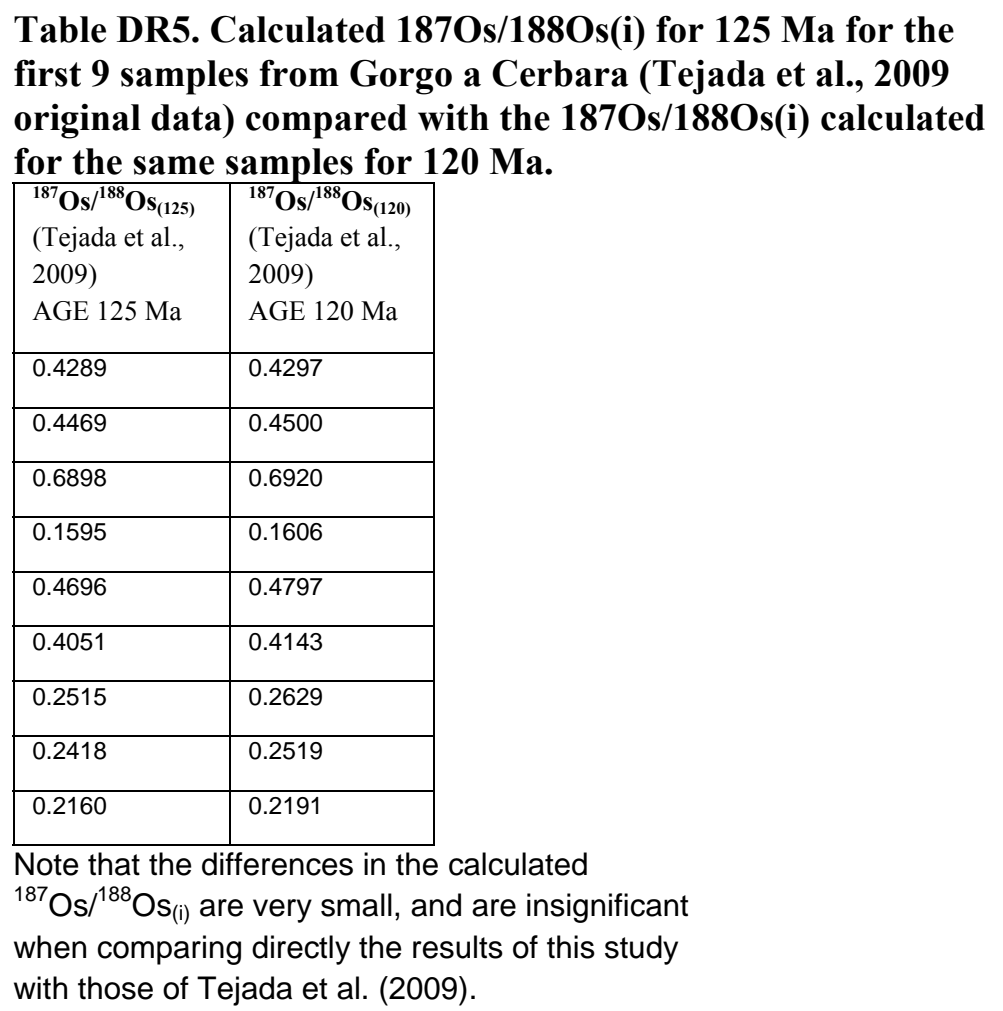

\section{References}

Bernoulli, D., Jenkyns, H.C., 2009. Ancient oceans and continental margins of the Alpine-Mediterranean Tethys: deciphering clues from Mesozoic pelagic sediments and ophiolites. Sedimentology, v. 56, p. 149-190.

Cohen, A.S., Waters, F.G., 1996. Separation of osmium from geological materials by solvent extraction for analysis by thermal ionisation mass spectrometry. Analytica Chimica Acta v. 332, p. 269-275.

Cohen, A.S., Coe, A.L., Harding, S.M., Schwark, L., 2004, Osmium isotope evidence for the regulation of atmospheric $\mathrm{CO}_{2}$ by continental weathering: Geology, v. 32, p. 157-160.

Erba, E., 1994, Nannofossils and superplumes: The early Aptian "nannoconid crisis". Paleoceanography, v. 9, p. 483-501.

Erba, E., Larson, R., 1998. The Cismon Apticore (Southern Alps, Italy): "Reference section" for the Lower Cretaceous at low latitudes. Riv. Ital. Paleontol. Stratigr., v. 104, p. 181-192.

Erba, E., Channell, J.E.T., Claps, M., Jones, C., Larson, R.L., Opdyke, B., Premoli Silva, I., Riva, A., Salvini, G., Torricelli, S., 1999, Integrated stratigraphy of the Cismon APTICORE (Southern Alps, Italy): a reference section for the Barremian-Aptian interval at low latitudes: J. Foraminifer. Res., v. 29, p. 371-391.

Erba, E., Bottini, C., Weissert, J.H., Keller, C.E., 2010, Calcareous Nannoplankton response to surfacewater acidification around Oceanic Anoxic Event 1a: Science, v. 329, p. 428-432.

Hay, W.W., 2009, Cretaceous Oceans and Ocean Modeling, In: Cretaceous Oceanic Red Beds: Stratigraphy, Composition, Origins, and Paleoceanographic and Paleoclimatic Significance, SEPM Spec. Pub. v. 91, p. 243-271. 
Ludwig, K.R., 1998, ISOPLOT: A Plotting and Regression Program for Radiogenic-Isotope Data, version 2.96 .

Malinverno, A., Erba, E., Herbert, T.D., 2010, Orbital tuning as an inverse problem: Chronology of the early Aptian oceanic anoxic event 1a (Selli Level) in the Cismon APTICORE: Paleoceanography, v. 25, PA2203, doi: 10.1029/2009PA001769.

Méhay, S., Keller, C.E., Bernasconi, S.M., Weissert, H., Erba, E., Bottini, C., Hochuli, P.A., 2009, A volcanic $\mathrm{CO}_{2}$ pulse triggered the Cretaceous Oceanic Anoxic Event 1a and a biocalcification crisis: Geology, v. 37, p. 819-822.

Mélières, F., Deroo, G., Herbin, J.P., 1978. Organic-matter-rich and hypersiliceous Aptian sediments from western Mid-Pacific Mountains. Deep Sea Drilling Project Leg 62, Initial Rep. Deep Sea Drill. Proj., v. 62, p. 903-915.

Ravizza, G., Norris, R.N., Blusztajn, J., Aubry, M.P., 2001, An osmium isotope excursion associated with the late Paleocene thermal maximum: Evidence of intensified chemical weathering:

Paleoceanography, v. 16, p. 155-163.

Roth, P.H., 1981. Mid-Cretaceous calcareous nannoplankton from central Pacific: implications for palaeoceanography. In Initial Reports of the Deep Sea Drilling Project 62 (J. Thiede, T. L. Vallier et al.), p. 471-89. Washington: U.S. Government Printing House.

Roy-Barman, M. and Allegre, C.J., 1995, ${ }^{187} \mathrm{Os} /{ }^{186}$ Os in oceanic island basalt: Tracing oceanic crust recycling in the mantle. Earth Planet. Sci. Lett., v. 129, p. 145.

Suan, G., Pittet, B., Bour, I., Mattioli, E., Duarte, L.V., Mailliot, S., 2008, Duration of the Early Toarcian carbon isotope excursion deduced from spectral analyses: Consequence for its possible causes: Earth and Planetary Science Letters, v. 267, p. 666-679.

Tejada, M.L.G., Katsuhiko S., Kuroda, J., Coccioni, R., Mahoney, J.J., Ohkouchi, N., Sakamoto, T., Tatsumi, Y., 2009, Ontong Java Plateau eruption as a trigger for the early Aptian oceanic anoxic event: Geology, v. 37, p. 855-858.

Thiede, J., Dean, W.E., Rea, D.K., Vallier, T.L., Adelseck C.G., 1981. The geologic history of the MidPacific Mountains in the central North Pacific Ocean: A synthesis of deep-sea drilling studies. Iniz. Rep. Deep Sea Drill. Proj. v. 62, p. 1073-1120.

Turgeon, S.C. and Creaser, R.A., 2008, Cretaceous oceanic anoxic event 2 triggered by a massive magmatic episode: Nature, v. 454, p. 323-326.

Voigt, S., Erbacher, J., Mutterlose, J., Weiss, W., Westerhold, T., Wiese, F., Wilmsen, M., Wonik, T., 2008, The Cenomanian-Turonian of the Wunstorf section-(north Germany): Global stratigraphic reference section and new orbital time scale for oceanic anoxic event 2: Newsl. Stratigr., v. 43, p. 6589, doi: 10.1127/0078-0421/2008/0043-0065.

Weissert, H., Lini, A., 1991. Ice Age interludes during the time of Cretaceous greenhouse climate? In Controversies in Modern Geology. Edited by D.W. Muller, J.A. McKenzie and H. Weissert, Academic, San Diego, Calif., p. 173-191.

Zachos, J.C. et al., 2005, Rapid acidification of the Ocean during the Paleocene-Eocene Thermal Maximum: Science, v. 308, p. 1611. 